Article

\title{
Convolution and Partial Sums of Certain Multivalent Analytic Functions Involving Srivastava-Tomovski Generalization of the Mittag-Leffler Function
}

\author{
Yi-Hui $X u^{1}$ and Jin-Lin Liu ${ }^{2, *}$ \\ 1 Department of Mathematics, Suqian College, Suqian 223800, China; yuanziqixu@126.com \\ 2 Department of Mathematics, Yangzhou University, Yangzhou 225002, China \\ * Correspondence: jlliu@yzu.edu.cn
}

Received: 7 October 2018; Accepted: 23 October 2018; Published: 5 November 2018

\begin{abstract}
We derive several properties such as convolution and partial sums of multivalent analytic functions associated with an operator involving Srivastava-Tomovski generalization of the Mittag-Leffler function.
\end{abstract}

Keywords: analytic function; Hadamard product (convolution); partial sum; Srivastava-Tomovski generalization of Mittag-Leffler function; subordination

\section{Introduction}

The Mittag-Leffler function $E_{\alpha}(z)$ [1] and its generalization $E_{\alpha, \beta}(z)$ [2] are defined by the following series:

$$
E_{\alpha}(z)=\sum_{n=0}^{\infty} \frac{z^{n}}{\Gamma(\alpha n+1)} \quad(z, \alpha \in \mathbb{C} ; \operatorname{Re}(\alpha)>0)
$$

and

$$
E_{\alpha, \beta}(z)=\sum_{n=0}^{\infty} \frac{z^{n}}{\Gamma(\alpha n+\beta)} \quad(z, \alpha, \beta \in \mathbb{C} ; \operatorname{Re}(\alpha)>0),
$$

respectively. It is known that these functions are extensions of exponential, hyperbolic, and trigonometric functions, since

$$
\begin{gathered}
E_{1}(z)=E_{1,1}(z)=e^{z} \\
E_{2}\left(z^{2}\right)=E_{2,1}\left(z^{2}\right)=\cosh z
\end{gathered}
$$

and

$$
E_{2}\left(-z^{2}\right)=E_{2,1}\left(-z^{2}\right)=\cos z .
$$

The functions $E_{\alpha}(z)$ and $E_{\alpha, \beta}(z)$ arise naturally in the resolvent of fractional integro-differential and fractional differential equations which are involved in random walks, super-diffusive transport problems, the kinetic equation, Lévy flights, and in the study of complex systems. In particular, the Mittag-Leffler function is an explicit formula for the solution the Riemann-Liouville fractional integrals that was developed by Hille and Tamarkin.

In [3], Srivastava and Tomovski defined a generalized Mittag-Leffler function $E_{\alpha, \beta}^{\gamma, k}(z)$ as follows:

$$
\begin{gathered}
E_{\alpha, \beta}^{\gamma, k}(z)=\sum_{n=0}^{\infty} \frac{(\gamma)_{n k} z^{n}}{\Gamma(\alpha n+\beta) n !}, \\
(\alpha, \beta, \gamma, k, z \in \mathbb{C} ; \quad \operatorname{Re}(\alpha)>\max \{0, \operatorname{Re}(k)-1\} ; \quad \operatorname{Re}(k)>0),
\end{gathered}
$$


where $(x)_{n}$ is the Pochhammer symbol

$$
(x)_{n}=\frac{\Gamma(x+n)}{\Gamma(x)}=x(x+1) \cdots(x+n-1) \quad(n \in \mathbb{N} ; \quad x \in \mathbb{C})
$$

and $(x)_{0}=1$. They proved that the function $E_{\alpha, \beta}^{\gamma, k}(z)$ given by (3) is an entire function in the complex plane. Recently, Attiya [4] proved that, if $\operatorname{Re}(\alpha) \geq 0$ with $\operatorname{Re}(k)=1$ and $\beta \neq 0$, the power series in (3) converges absolutely and analytically in $\mathbb{U}=\{z:|z|<1\}$ for all $\gamma \in \mathbb{C}$. We call the function $E_{\alpha, \beta}^{\gamma, k}(z)$ the Srivastava-Tomovski generalization of the Mittag-Leffler function.

Let $\mathcal{A}(p)$ be the class of functions of the form

$$
f(z)=z^{p}+\sum_{n=2}^{\infty} a_{n+p-1} z^{n+p-1} \quad(p \in \mathbb{N})
$$

which are analytic in $\mathbb{U}$. For $p=1$, we write $\mathcal{A}:=\mathcal{A}(1)$. The Hadamard product (or convolution) of two functions

$$
f_{j}(z)=z^{p}+\sum_{n=2}^{\infty} a_{n+p-1, j} z^{n+p-1} \in \mathcal{A}(p) \quad(j=1,2)
$$

is given by

$$
\left(f_{1} * f_{2}\right)(z)=z^{p}+\sum_{n=2}^{\infty} a_{n+p-1,1} a_{n+p-1,2} z^{n+p-1}=\left(f_{2} * f_{1}\right)(z) .
$$

Let $\mathcal{P}$ denote the class of functions $\varphi$ with $\varphi(0)=1$. Suppose that $f$ and $g$ are analytic in $\mathbb{U}$. If there exists a Schwarz function $w$ such that $f(z)=g(w(z))$ for $z \in \mathbb{U}$, then we say that the function $f$ is subordinate to $g$ and write $f(z) \prec g(z)$ for $z \in \mathbb{U}$. Furthermore, if $g$ is univalent in $\mathbb{U}$, then the following equivalence holds true:

$$
f(z) \prec g(z) \quad(z \in \mathbb{U}) \Leftrightarrow f(0)=g(0) \quad \text { and } \quad f(\mathbb{U}) \subset g(\mathbb{U}) .
$$

Throughout this paper, we assume that

$$
\alpha, \beta, \gamma, k \in \mathbb{C} ; \quad \operatorname{Re}(\alpha)>\max \{0, \operatorname{Re}(k)-1\} \quad \text { and } \operatorname{Re}(k)>0 .
$$

We define the function $Q_{\alpha, \beta}^{\gamma, k}(z) \in \mathcal{A}(p)$ associated with the Srivastava-Tomovski generalization of the Mittag-Leffler function by

$$
Q_{\alpha, \beta}^{\gamma, k}(z)=\frac{\Gamma(\alpha+\beta)}{(\gamma)_{k}} z^{p-1}\left(E_{\alpha, \beta}^{\gamma, k}(z)-\frac{1}{\Gamma(\beta)}\right) \quad(z \in \mathbb{U}) .
$$

For $f \in \mathcal{A}(p)$, we introduce a new operator $H_{\alpha, \beta}^{\gamma, k}: \mathcal{A}(p) \rightarrow \mathcal{A}(p)$ by

$$
\begin{aligned}
H_{\alpha, \beta}^{\gamma, k} f(z) & =Q_{\alpha, \beta}^{\gamma, k}(z) * f(z) \\
& =z^{p}+\sum_{n=2}^{\infty} \frac{\Gamma(\gamma+n k) \Gamma(\alpha+\beta)}{\Gamma(\gamma+k) \Gamma(\alpha n+\beta) n !} a_{n+p-1} z^{n+p-1} .
\end{aligned}
$$

Note that $H_{0, \beta}^{1,1} f(z)=f(z)$. From (6), we easily have the following identity:

$$
z\left(H_{\alpha, \beta}^{\gamma, k} f(z)\right)^{\prime}=\left(\frac{\gamma}{k}+1\right) H_{\alpha, \beta}^{\gamma+1, k} f(z)-\left(\frac{\gamma}{k}+1-p\right) H_{\alpha, \beta}^{\gamma, k} f(z) .
$$

It is noteworthy to mention that the Fox-Wright hypergeometric function ${ }_{q} \Psi_{S}$ is more general than many of the extensions of the Mittag-Leffler function.

Now, we introduce a new subclass of $\mathcal{A}(p)$ by using the operator $H_{\alpha, \beta}^{\gamma, k}$. 
Definition 1. A function $f \in \mathcal{A}(p)$ is said to be in $\Omega_{\alpha, \beta}^{\gamma, k}(\lambda ; \varphi)$ if it satisfies the first-order differential subordination:

$$
(1-\lambda) z^{-p} H_{\alpha, \beta}^{\gamma, k} f(z)+\frac{\lambda}{p} z^{-p+1}\left(H_{\alpha, \beta}^{\gamma, k} f(z)\right)^{\prime} \prec \varphi(z),
$$

where $\lambda \in \mathbb{C}$ and $\varphi \in \mathcal{P}$.

Lemma 1. ([5]). Let $g(z)=1+\sum_{n=m}^{\infty} b_{n} z^{n}(m \in \mathbb{N})$ be analytic in $\mathbb{U}$. If $\operatorname{Re}(g(z))>0(z \in \mathbb{U})$, then

$$
\operatorname{Re}(g(z)) \geq \frac{1-|z|^{m}}{1+|z|^{m}} \quad(z \in \mathbb{U})
$$

The study of the Mittag-Leffler function is an interesting topic in Geometric Function Theory. Many properties of the Mittag-Leffler function and the generalized Mittag-Leffler function can be found, e.g., in [6-22]. In this paper we shall make a further contribution to the subject by showing some interesting properties such as convolution and partial sums for functions in the class $\Omega_{\alpha, \beta}^{\gamma, k}(\lambda ; \varphi)$.

\section{Properties of the Class $\Omega_{\alpha, \beta}^{\gamma, k}(\lambda ; \varphi)$}

Theorem 1. Let $\lambda \geq 0$ and

$$
f_{j}(z)=z^{p}+\sum_{n=2}^{\infty} a_{n+p-1, j} z^{n+p-1} \in \Omega_{\alpha, \beta}^{\gamma, k}\left(\lambda ; \varphi_{j}\right) \quad(j=1,2),
$$

where

$$
\varphi_{j}(z)=\frac{1+A_{j} z}{1+B_{j} z} \quad \text { and } \quad-1 \leq B_{j}<A_{j} \leq 1 .
$$

If $f \in \mathcal{A}(p)$ is defined by

$$
H_{\alpha, \beta}^{\gamma, k} f(z)=\left(H_{\alpha, \beta}^{\gamma, k} f_{1}(z)\right) *\left(H_{\alpha, \beta}^{\gamma, k} f_{2}(z)\right)
$$

then $f \in \Omega_{\alpha, \beta}^{\gamma, k}(\lambda ; \varphi)$, where

$$
\varphi(z)=\rho+(1-\rho) \frac{1+z}{1-z}
$$

and $\rho$ is given by

$$
\rho= \begin{cases}1-\frac{4\left(A_{1}-B_{1}\right)\left(A_{2}-B_{2}\right)}{\left(1-B_{1}\right)\left(1-B_{2}\right)}\left(1-\frac{p}{\lambda} \int_{0}^{1} \frac{t \frac{p}{\lambda}-1}{1+t} d t\right) & (\lambda>0), \\ 1-\frac{2\left(A_{1}-B_{1}\right)\left(A_{2}-B_{2}\right)}{\left(1-B_{1}\right)\left(1-B_{2}\right)} & (\lambda=0) .\end{cases}
$$

The bound $\rho$ is sharp when $B_{1}=B_{2}=-1$.

Proof. We consider the case when $\lambda>0$. Since $f_{j} \in \Omega_{\alpha, \beta}^{\gamma, k}\left(\lambda ; \varphi_{j}\right)$, it follows that

$$
\begin{aligned}
p_{j}(z) & =(1-\lambda) z^{-p} H_{\alpha, \beta}^{\gamma, k} f_{j}(z)+\frac{\lambda}{p} z^{-p+1}\left(H_{\alpha, \beta}^{\gamma, k} f_{j}(z)\right)^{\prime} \\
& \prec \frac{1+A_{j} z}{1+B_{j} z} \quad(j=1,2)
\end{aligned}
$$


and

$$
\begin{aligned}
H_{\alpha, \beta}^{\gamma, k} f_{j}(z) & =\frac{p}{\lambda} z^{-\frac{p(1-\lambda)}{\lambda}} \int_{0}^{z} t^{\frac{p}{\lambda}-1} p_{j}(t) d t \\
& =\frac{p}{\lambda} z^{p} \int_{0}^{1} t^{\frac{p}{\lambda}-1} p_{j}(t z) d t \quad(j=1,2) .
\end{aligned}
$$

Now, if $f \in \mathcal{A}(p)$ is defined by (11), we find from (14) that

$$
\begin{aligned}
H_{\alpha, \beta}^{\gamma, k} f(z) & =\left(H_{\alpha, \beta}^{\gamma, k} f_{1}(z)\right) *\left(H_{\alpha, \beta}^{\gamma, k} f_{2}(z)\right) \\
& =\left(\frac{p}{\lambda} z^{p} \int_{0}^{1} t^{\frac{p}{\lambda}-1} p_{1}(t z) d t\right) *\left(\frac{p}{\lambda} z^{p} \int_{0}^{1} t^{\frac{p}{\lambda}-1} p_{2}(t z) d t\right) \\
& =\frac{p}{\lambda} z^{p} \int_{0}^{1} t^{\frac{p}{\lambda}-1} p_{0}(t z) d t,
\end{aligned}
$$

where

$$
p_{0}(z)=\frac{p}{\lambda} \int_{0}^{1} t^{\frac{p}{\lambda}-1}\left(p_{1} * p_{2}\right)(t z) d t .
$$

Further, by using (14) and the Herglotz theorem, we see that

$$
\operatorname{Re}\left\{\left(\frac{p_{1}(z)-\rho_{1}}{1-\rho_{1}}\right) *\left(\frac{1}{2}+\frac{p_{2}(z)-\rho_{2}}{2\left(1-\rho_{2}\right)}\right)\right\}>0 \quad(z \in \mathbb{U}),
$$

which leads to

$$
\operatorname{Re}\left\{\left(p_{1} * p_{2}\right)(z)\right\}>\rho_{0}=1-2\left(1-\rho_{1}\right)\left(1-\rho_{2}\right) \quad(z \in \mathbb{U}),
$$

where

$$
0 \leq \rho_{j}=\frac{1-A_{j}}{1-B_{j}}<1 \quad(j=1,2) .
$$

Moreover, according to Lemma, we have

$$
\operatorname{Re}\left\{\left(p_{1} * p_{2}\right)(z)\right\} \geq \rho_{0}+\left(1-\rho_{0}\right) \frac{1-|z|}{1+|z|} \quad(z \in \mathbb{U}) .
$$

Thus, it follows from (16) to (18) that

$$
\begin{aligned}
& \operatorname{Re}\left\{(1-\lambda) z^{-p} H_{\alpha, \beta}^{\gamma, k} f(z)+\frac{\lambda}{p} z^{-p+1}\left(H_{\alpha, \beta}^{\gamma, k} f(z)\right)^{\prime}\right\}=\operatorname{Re}\left\{p_{0}(z)\right\} \\
& =\frac{p}{\lambda} \int_{0}^{1} t^{\frac{p}{\lambda}-1} \operatorname{Re}\left\{\left(p_{1} * p_{2}\right)(t z)\right\} d t \\
& \geq \frac{p}{\lambda} \int_{0}^{1} t^{\frac{p}{\lambda}-1}\left(\rho_{0}+\left(1-\rho_{0}\right) \frac{1-|z| t}{1+|z| t}\right) d t \\
& >\rho_{0}+\frac{p\left(1-\rho_{0}\right)}{\lambda} \int_{0}^{1} t^{\frac{p}{\lambda}-1} \frac{1-t}{1+t} d t \\
& =1-4\left(1-\rho_{1}\right)\left(1-\rho_{2}\right)\left(1-\frac{p}{\lambda} \int_{0}^{1} \frac{t^{\frac{p}{\lambda}-1}}{1+t} d t\right) \\
& =\rho,
\end{aligned}
$$

which proves that $f \in \Omega_{\alpha, \beta}^{\gamma, k}(\lambda ; \varphi)$ for the function $\varphi$ given by (12).

In order to show that the bound $\rho$ is sharp, we take the functions $f_{j} \in \mathcal{A}(p)(j=1,2)$ defined by

$$
H_{\alpha, \beta}^{\gamma, k} f_{j}(z)=\frac{p}{\lambda} z^{-\frac{p(1-\lambda)}{\lambda}} \int_{0}^{z} t^{\frac{p}{\lambda}-1}\left(\frac{1+A_{j} t}{1-t}\right) d t \quad(j=1,2),
$$


for which we have

$$
\begin{aligned}
p_{j}(z) & =(1-\lambda) z^{-p} H_{\alpha, \beta}^{\gamma, k} f_{j}(z)+\frac{\lambda}{p} z^{-p+1}\left(H_{\alpha, \beta}^{\gamma, k} f_{j}(z)\right)^{\prime} \\
& =\frac{1+A_{j} z}{1-z} \quad(j=1,2)
\end{aligned}
$$

and

$$
\begin{aligned}
\left(p_{1} * p_{2}\right)(z) & =\frac{1+A_{1} z}{1-z} * \frac{1+A_{2} z}{1-z} \\
& =1-\left(1+A_{1}\right)\left(1+A_{2}\right)+\frac{\left(1+A_{1}\right)\left(1+A_{2}\right)}{1-z} .
\end{aligned}
$$

Hence, for the function $f$ given by (11), we have

$$
\begin{aligned}
& (1-\lambda) z^{-p} H_{\alpha, \beta}^{\gamma, k} f(z)+\frac{\lambda}{p} z^{-p+1}\left(H_{\alpha, \beta}^{\gamma, k} f(z)\right)^{\prime} \\
& =\frac{p}{\lambda} \int_{0}^{1} t^{\frac{p}{\lambda}-1}\left(1-\left(1+A_{1}\right)\left(1+A_{2}\right)+\frac{\left(1+A_{1}\right)\left(1+A_{2}\right.}{1-t z}\right) d t \\
& \rightarrow \rho \quad(\text { as } \quad z \rightarrow-1),
\end{aligned}
$$

which shows that the number $\rho$ is the best possible when $B_{1}=B_{2}=-1$.

For the case when $\lambda=0$, the proof of Theorem 1 is simple, and we choose to omit the details involved. Now the proof of Theorem 1 is completed.

Theorem 2. Let $\alpha, \beta, \gamma, k$, and $\lambda$ be positive real numbers. Let $f(z)=z^{p}+\sum_{n=2}^{\infty} a_{n+p-1} z^{n+p-1} \in \mathcal{A}(p)$, $s_{1}(z)=z^{p}$, and $s_{m}(z)=z^{p}+\sum_{n=2}^{m} a_{n+p-1} z^{n+p-1}(m \geq 2)$. Suppose that

$$
\sum_{n=2}^{\infty} c_{n}\left|a_{n+p-1}\right| \leq 1
$$

where

$$
c_{n}=\frac{1-B}{A-B} \cdot \frac{\Gamma(\gamma+n k) \Gamma(\alpha+\beta)}{\Gamma(\beta+n \alpha) \Gamma(\gamma+k) n !}\left(1+\frac{\lambda}{p}(n-1)\right)
$$

and $-1 \leq B<A \leq 1$.

(i) If $-1 \leq B \leq 0$, then $f \in \Omega_{\alpha, \beta}^{\gamma, k}\left(\lambda ; \frac{1+A z}{1+B z}\right)$.

(ii) If $\left\{c_{n}\right\}_{1}^{\infty}$ is nondecreasing, then

$$
\operatorname{Re}\left\{\frac{f(z)}{s_{m}(z)}\right\}>1-\frac{1}{c_{m+1}}
$$

and

$$
\operatorname{Re}\left\{\frac{s_{m}(z)}{f(z)}\right\}>\frac{c_{m+1}}{1+c_{m+1}}
$$

for $z \in \mathbb{U}$. The estimates in (22) and (23) are sharp for each $m \in \mathbb{N}$.

Proof. From the assumptions of Theorem 2, we have $c_{n}>0(n \in \mathbb{N})$. Let

$$
\begin{aligned}
J(z) & =(1-\lambda) z^{-p} H_{\alpha, \beta}^{\gamma, k} f(z)+\frac{\lambda}{p} z^{-p+1}\left(H_{\alpha, \beta}^{\gamma, k} f(z)\right)^{\prime} \\
& =1+\sum_{n=2}^{\infty} \frac{\Gamma(\gamma+n k) \Gamma(\alpha+\beta)}{\Gamma(\beta+n \alpha) \Gamma(\gamma+k) n !}\left(1+\frac{\lambda}{p}(n-1)\right) a_{n+p-1} z^{n-1} .
\end{aligned}
$$


(i) For $-1 \leq B \leq 0$ and $z \in \mathbb{U}$, it follows from (20), (21), and (24), that

$$
\begin{aligned}
& \left|\frac{J(z)-1}{A-B J(z)}\right| \\
& =\left|\frac{\sum_{n=2}^{\infty} \frac{\Gamma(\gamma+n k) \Gamma(\alpha+\beta)}{\Gamma(\beta+n \alpha) \Gamma(\gamma+k) n !}\left(1+\frac{\lambda}{p}(n-1)\right) a_{n+p-1} z^{n-1}}{A-B-B \sum_{n=2}^{\infty} \frac{\Gamma(\gamma+n k) \Gamma(\alpha+\beta)}{\Gamma(\beta+n \alpha) \Gamma(\gamma+k) n !}\left(1+\frac{\lambda}{p}(n-1)\right) a_{n+p-1} z^{n-1}}\right| \\
& \leq \frac{\sum_{n=2}^{\infty} c_{n}\left|a_{n+p-1}\right|}{1-B+B \sum_{n=2}^{\infty} c_{n}\left|a_{n+p-1}\right|} \leq 1,
\end{aligned}
$$

which implies that

$$
(1-\lambda) z^{-p} H_{\alpha, \beta}^{\gamma, k} f(z)+\frac{\lambda}{p} z^{-p+1}\left(H_{\alpha, \beta}^{\gamma, k} f(z)\right)^{\prime} \prec \frac{1+A z}{1+B z} .
$$

Hence, $f \in \Omega_{\alpha, \beta}^{\gamma, k}\left(\lambda ; \frac{1+A z}{1+B z}\right)$.

(ii) Under the hypothesis in part (ii) of Theorem 2, we can see from (21) that $c_{n+1}>c_{n}>1$ $(n \in \mathbb{N})$. Therefore, we have

$$
\sum_{n=2}^{m}\left|a_{n+p-1}\right|+c_{m+1} \sum_{n=m+1}^{\infty}\left|a_{n+p-1}\right| \leq \sum_{n=2}^{\infty} c_{n}\left|a_{n+p-1}\right| \leq 1 .
$$

Upon setting

$$
p_{1}(z)=c_{m+1}\left\{\frac{f(z)}{s_{m}(z)}-\left(1-\frac{1}{c_{m+1}}\right)\right\}=1+\frac{c_{m+1} \sum_{n=m+1}^{\infty} a_{n+p-1} z^{n-1}}{1+\sum_{n=2}^{\infty} a_{n+p-1} z^{n-1}},
$$

and applying (25), we find that

$$
\left|\frac{p_{1}(z)-1}{p_{1}(z)+1}\right| \leq \frac{c_{m+1} \sum_{n=m+1}^{\infty}\left|a_{n+p-1}\right|}{2-2 \sum_{n=2}^{m}\left|a_{n+p-1}\right|-c_{m+1} \sum_{n=m+1}^{\infty}\left|a_{n+p-1}\right|} \leq 1 \quad(z \in \mathbb{U}),
$$

which readily yields (22).

If we take

$$
f(z)=z^{p}-\frac{z^{m+p}}{c_{m+1}}
$$

then

$$
\frac{f(z)}{s_{m}(z)}=1-\frac{z^{m}}{c_{m+1}} \rightarrow 1-\frac{1}{c_{m+1}} \quad \text { and } \quad z \rightarrow 1^{-},
$$

which shows that the bound in (22) is the best possible for each $m \in \mathbb{N}$.

Similarly, if we put

$$
p_{2}(z)=\left(1+c_{m+1}\right)\left(\frac{s_{m}(z)}{f(z)}-\frac{c_{m+1}}{1+c_{m+1}}\right),
$$

then we can deduce that

$$
\begin{aligned}
\left|\frac{p_{2}(z)-1}{p_{2}(z)+1}\right| & \leq \frac{\left(1+c_{m+1}\right) \sum_{n=m+1}^{\infty}\left|a_{n+p-1}\right|}{2-2 \sum_{n=2}^{m}\left|a_{n+p-1}\right|-\left(c_{m+1}-1\right) \sum_{n=m+1}^{\infty}\left|a_{n+p-1}\right|} \\
& \leq 1 \quad(z \in \mathbb{U}),
\end{aligned}
$$

which yields (23).

The bound in (23) is sharp for each $m \in \mathbb{N}$, with the extremal function $f$ given by (26). The proof of Theorem 2 is thus completed. 
Author Contributions: All authors contributed equally.

Funding: This research is supported by National Natural Science Foundation of China (Grant No. 11571299) and Natural Science Foundation of Jiangsu Gaoxiao (Grant No. 17KJB110019).

Acknowledgments: The authors would like to express sincere thanks to the referees for careful reading and suggestions which helped us to improve the paper.

Conflicts of Interest: The authors declare no conflict of interest.

\section{References}

1. Mittag-Leffler, G.M. Sur la nouvelle fonction $E(x)$. C. R. Acad. Sci. Paris 1903, 137, 554-558.

2. Wiman, A. Über den Fundamental satz in der Theorie der Funcktionen E(x). Acta Math. 1905, $29,191-201$. [CrossRef]

3. Srivastava, H.M.; Tomovski, Ž. Fractional calculus with an integral operator containing a generalized Mittag-Leffler function in the kernal. Appl. Math. Comput. 2009, 211, 198-210.

4. Attiya, A.A. Some applications of Mittag-Leffler function in the unit disk. Filomat 2016, 30, $2075-2081$. [CrossRef]

5. MacGregor, T.H. Functions whose derivative has a positive real part. Trans. Am. Math. Soc. 1962, 104, 532-537. [CrossRef]

6. Tomovski, Z. Generalized Cauchy type problems for nonlinear fractional differential equations with composite fractional derivative operator. Nonlinear Anal. 2012, 75, 3364-3384. [CrossRef]

7. Tomovski, Z.; Hilfer, R.; Srivastava, H.M. Fractional and operational calculus with generalized fractional derivative operators and Mittag-Leffler type functions. Integr. Transf. Spec. Funct. 2010, 21, 797-814. [CrossRef]

8. Bansal, D.; Prajapat, J.K. Certain geometric properties of the Mittag-Leffler functions. Complex Var. Elliptic Eq. 2016, 61, 338-350. [CrossRef]

9. Grag, M.; Manohar, P.; Kalla, S.L. A Mittag-Leffler-type function of two variables. Integral Transf. Spec. Funct. 2013, 24, 934-944. [CrossRef]

10. Srivastava, H.M.; Bansal, D. Close-to-convexity of a certain family of $q$-Mittag-Leffler functions. J. Nonlinear Var. Anal. 2017, 1, 61-69.

11. Srivastava, H.M.; Frasin, B.A.; Pescar, V. Univalence of integral operators involving Mittag-Leffler functions. Appl. Math. Inf. Sci. 2017, 11, 635-641. [CrossRef]

12. Liu, J.-L. Notes on Jung-Kim-Srivastava integral operator. J. Math. Anal. Appl. 2004, 294, 96-103. [CrossRef]

13. Assante, D.; Cesarano, C.; Fornaro, C.; Vazquez, L. Higher order and fractional diffusive equations. J. Eng. Sci. Technol. Rev. 2015, 8, 202-204. [CrossRef]

14. Cesarano, C.; Fornaro, C.; Vazquez, L. A note on a special class of hermite polynomials. Int. J. Pure Appl. Math. 2015, 98, 261-273. [CrossRef]

15. Kapoor, G.P.; Mishra, A.K. Coefficient estimates for inverses of starlike functions of positive order. J. Math. Anal. Appl. 2007, 329, 922-934. [CrossRef]

16. Ma, W.C.; Minda, D. A unified treatment of some special classes of univalent functions. In Proceedings of the Conference on Complex Analysis, Tianjin, China, 18-23 August 1992; pp. 157-169.

17. Marin, M.; Florea, O. On temporal behavior of solutions in thermoelasticity of porous micropolar bodies. An. St. Univ. Ovidius Constanta-Seria Math. 2014, 22, 169-188.

18. Miller, S.S.; Mocanu, P.T. Differential subordinations and univalent functions. Mich. Math. J. 1981, 28, 157-171. [CrossRef]

19. Nishiwaki, J.; Owa, S. Coefficient inequalities for analytic functions. Int. J. Math. Math. Sci. 2002, 29, $285-290$. [CrossRef]

20. Ruscheweyh, S. Convolutions in Geometric Function Theory; Les Presses de 1'Université de Montréal: Montréal, QC, Canada, 1982. 
21. Seoudy, T.M.; Aouf, M.K. Coefficient estimates of new classes of $q$-starlike and $q$-convex functions of complex order. J. Math. Inequal. 2016, 10, 135-145. [CrossRef]

22. Srivastava, H.M. Some Fox-Wright generalized hypergeometric functions and associated families of convolution operators. Appl. Anal. Discret. Math. 2007, 1, 56-71. 